\title{
Dyslexia and language impairment associated genetic markers influence cortical thickness and white matter in typically developing children
}

\author{
John D. Eicher ${ }^{1}$ • Angela M. Montgomery ${ }^{2}$ - Natacha Akshoomoff ${ }^{3,4}$. \\ David G. Amaral ${ }^{5}$ • Cinnamon S. Bloss ${ }^{6}$ • Ondrej Libiger ${ }^{6}$ - Nicholas J. Schork ${ }^{6}$ • \\ Burcu F. Darst ${ }^{6}$ - B. J. Casey ${ }^{7} \cdot$ Linda Chang $^{8}$ • Thomas Ernst $^{8}$ • Jean Frazier ${ }^{9}$. \\ Walter E. Kaufmann ${ }^{10,11}$ - Brian Keating ${ }^{8}$ - Tal Kenet ${ }^{12}$ • David Kennedy ${ }^{9}$.

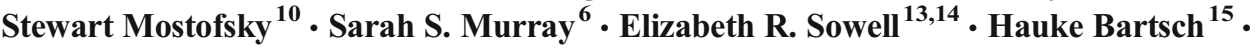 \\ Joshua M. Kuperman ${ }^{15,16}$ • Timothy T. Brown ${ }^{3,15,16}$ • Donald J. Hagler Jr. ${ }^{15,17}$. \\ Anders M. Dale ${ }^{4,15,16,17,18}$ • Terry L. Jernigan ${ }^{3,4,17,18}$ • Jeffrey R. Gruen ${ }^{1,2,19,20}$ • \\ on behalf of the Pediatric Imaging Neurocognition Genetics study
}

Published online: 9 May 2015

(C) The Author(s) 2015. This article is published with open access at Springerlink.com

\begin{abstract}
Dyslexia and language impairment (LI) are complex traits with substantial genetic components. We recently completed an association scan of the DYX2 locus, where we observed associations of markers in DCDC2, KIAA0319,
\end{abstract}

Electronic supplementary material The online version of this article (doi:10.1007/s11682-015-9392-6) contains supplementary material, which is available to authorized users.

Jeffrey R. Gruen

jeffrey.gruen@yale.edu

1 Department of Genetics, Yale University, New Haven, CT 06520, USA

2 Department of Pediatrics, Yale University School of Medicine, New Haven, CT 06520, USA

3 Center for Human Development, University of California, La Jolla, San Diego, CA 92037, USA

4 Department of Psychiatry, University of California, La Jolla, San Diego, CA 92037, USA

5 Department of Psychiatry and Behavioral Sciences, University of California, Davis, CA 95817, USA

6 Scripps Genomic Medicine, Scripps Health, Scripps Translational Science Institute, La Jolla, CA 92037, USA

7 Sackler Institute for Developmental Psychobiology, Weil Cornell Medical College, New York, NY 10065, USA

8 Department of Medicine, Queen's Medical Center, University of Hawaii, Honolulu, HI 96813, USA

9 Department of Psychiatry, University of Massachusetts Medical School, Boston, MA 01655, USA

10 Kennedy Krieger Institute, 707 N. Broadway, Baltimore, MD21205, USA
$A C O T 13$, and FAM65B with reading-, language-, and IQrelated traits. Additionally, the effects of reading-associated DYX3 markers were recently characterized using structural neuroimaging techniques. Here, we assessed the neuroimag-
11 Department of Neurology, Harvard Medical School, Children's Hospital Boston, Boston, MA 02115, USA

12 Department of Neurology and Athinoula A. Martinos Center for Biomedical Imaging, Massachusetts General Hospital, Charlestown, MA 02129, USA

13 Department of Pediatrics, University of Southern California, Los Angeles, CA 90027, USA

14 Developmental Cognitive Neuroimaging Laboratory Children's Hospital, Los Angeles, CA 90027, USA

15 Multimodal Imaging Laboratory, University of California, La Jolla, San Diego, CA 92037, USA

16 Department of Neurosciences, University of California, La Jolla, San Diego, CA 92037, USA

17 Radiology University of California, La Jolla, San Diego, CA 92037, USA

18 Cognitive Science University of California, La Jolla, San Diego, CA 92037, USA

19 Department of Investigative, School of Medicine, Medicine Yale University, New Haven, CT 06520, USA

20 Department of Pediatrics, Genetics, and Investigative Medicine, Yale Child Health Research Center, 464 Congress Avenue, New Haven, CT 06520-8081, USA 
ing implications of associated DYX2 and DYX3 markers, using cortical volume, cortical thickness, and fractional anisotropy. To accomplish this, we examined eight DYX2 and three DYX3 markers in 332 subjects in the Pediatrics Imaging Neurocognition Genetics study. Imaging-genetic associations were examined by multiple linear regression, testing for influence of genotype on neuroimaging. Markers in DYX2 genes KIAA0319 and FAM65B were associated with cortical thickness in the left orbitofrontal region and global fractional anisotropy, respectively. KIAAO319 and ACOT13 were suggestively associated with overall fractional anisotropy and left pars opercularis cortical thickness, respectively. DYX3 markers showed suggestive associations with cortical thickness and volume measures in temporal regions. Notably, we did not replicate association of DYX3 markers with hippocampal measures. In summary, we performed a neuroimaging follow-up of reading-, language-, and IQ-associated DYX2 and DYX3 markers. DYX2 associations with cortical thickness may reflect variations in their role in neuronal migration. Furthermore, our findings complement gene expression and imaging studies implicating DYX3 markers in temporal regions. These studies offer insight into where and how DYX2 and DYX3 risk variants may influence neuroimaging traits. Future studies should further connect the pathways to risk variants associated with neuroimaging/neurocognitive outcomes.

Keywords Dyslexia - Language impairment - KIAA0319 . DYX3 - DYX2 - Imaging-genetics

$\begin{array}{ll}\text { Abbreviations } \\ \text { RD } & \text { Reading disability } \\ \text { LI } & \text { Language impairment } \\ \text { SSD } & \text { Speech sound disorder } \\ \text { LD } & \text { Linkage disequilibrium } \\ \text { GWAS } & \text { Genome-wide association study } \\ \text { fMRI } & \text { Functional magnetic resonance imaging } \\ \text { SMRI } & \text { Structural magnetic resonance imaging } \\ \text { FA } & \text { Fractional anisotropy } \\ \text { DTI } & \text { Diffusion tensor imaging } \\ \text { PING } & \text { Pediatric imaging neurocognition genetics } \\ \text { UCSD } & \text { University of California at San Diego } \\ \text { ROI } & \text { Region of interest } \\ \text { GAF } & \text { Genetic ancestry factor } \\ \text { CC } & \text { Corpus callosum } \\ \text { SLF } & \text { Superior longitudinal fasciculus } \\ \text { tSLF } & \text { Temporal superior longitudinal fasciculus }\end{array}$

\section{Introduction}

Neurocognitive and language traits are complex phenotypes with substantial environmental and genetic components.
Specifically, dyslexia (also known as reading disability or $\mathrm{RD})$ and language impairment (LI) as well as quantitative performance in reading, language, and cognitive skills are heritable traits, with heritability estimates ranging from 45 to 84 \% (DeFries et al. 1987; Bishop and Hayiou-Thomas 2008; Pennington and Bishop 2009; van Soelen et al. 2011). RD is characterized by unexpected difficulties in reading despite normal child development as well as adequate educational instruction and opportunity (Pennington and Bishop 2009). Children with LI generally have unexplained difficulties in oral language, as opposed to written language deficits seen in RD, despite normal child development and adequate opportunity (Pennington and Bishop 2009; Newbury et al. 2010). Both RD and LI adversely affect a child's academic, linguistic, and social development and can hamper academic achievement.

Several candidate loci and genes for RD and LI have been identified. Two of these candidate regions are the DYX2 locus on chromosome 6p22 and the DYX3 locus on chromosome 2p12. Both DYX2 and DYX3 were identified through linkage studies of families with RD (Fagerheim et al. 1999; Anthoni et al. 2007; Kaminen et al. 2003; Cardon et al. 1994; Gayán et al. 1999; Kaplan et al. 2002; Deffenbacher et al. 2004). The DYX2 locus is the most replicated RD risk locus, with subsequent association studies identifying two well-established risk genes, DCDC2 and KIAA0319 (Meng et al. 2005; Schumacher et al. 2006; Harold et al. 2006). Following their associations with $\mathrm{RD}$, other studies have shown that genes within the DYX2 locus contribute to quantitative reading and language performance as well as other related neurocognitive and language traits including LI, overall cognition, and speech sound disorder (SSD) (Scerri et al. 2011; Powers et al. 2013; Eicher et al. 2014; Smith et al. 2005; Newbury et al. 2011). Our group recently reported the results of an association scan across the entire DYX2 locus with reading, language, and cognitive traits in an unselected, population-based sample from the United Kingdom (Powers et al. 2013; Eicher et al. 2014). There, we recapitulated the associations of $D C D C 2$ and KIAA0319 as well as implicated two new candidate genes FAM65B and CMAHP. Markers within ACOT13 (also known as THEM2) and C6orf62 also were associated with these traits but were in linkage disequilibrium (LD) with a previously identified risk haplotype in KIAA0319, leading us to hypothesize that the associations of ACOT13 and C6orf62 tagged variation in KIAA0319 (Eicher et al. 2014; Francks et al. 2004; Paracchini et al. 2006).

The DYX3 locus on chromosome $2 \mathrm{p} 12$ is less studied than the DYX2 locus. Two candidate genes, MRPL19 and GCFC2 (also referred to as $C 2$ orf 3 ), have been proposed with mixed results in replication analyses for each (Fagerheim et al. 1999; Anthoni et al. 2007; Kaminen et al. 2003; Peyrard-Janvid et al. 2004; Paracchini et al. 2011; Scerri et al. 2012). Similar to DYX2, the DYX3 locus appears to contribute not only to 
$\mathrm{RD}$ and reading-related traits but other neurocognitive domains and disorders. For instance, Scerri et al. found association of markers within the MRPL19/GCFC2 locus with both verbal and performance IQ (Scerri et al. 2012) in a populationbased European sample. Furthermore, a genome-wide association study (GWAS) showed a suggestive association of a marker in GCFC2 with Alzheimer's disease (Melville et al. 2012). Although not exactly related to pediatric language disorders, this suggestive association with Alzheimer's disease, a late-onset disorder with neurocognitive impairments, further implicates a possible general role of $G C F C 2$ in overall neurocognitive skills as well as language-related domains.

These studies have largely focused on the genetic relationship with neurobehavioral measures of reading, language, and cognition, as these are the instruments used clinically. However, solely using neurobehavioral measures does not necessarily give insight into the underlying molecular and neurological mechanisms of these traits. Intermediate phenotypes, that represent biological phenomena closer to the genetic function, can provide a powerful approach to gain insight into pathophysiology (Thompson et al. 2010). Therefore, to gain further insight into neurological mechanism, in vivo neuroimaging techniques can reveal structural, connectivity, and functional implications of genes and associated genetic variants (Eicher and Gruen 2013; Graham and Fisher 2013).

In this vein, human imaging-genetics studies have examined the relationships of DYX2 and DYX3 risk variants with various neuroimaging modalities. $D C D C 2$ variants have been associated with overall grey matter volume as well as in superior prefrontal, temporal, and occipital networks (Meda et al. 2008; Jamadar et al. 2011). KIAA0319 markers were also associated with gray matter in the superior and inferior cerebellar networks (Jamadar et al. 2011). Darki et al. reported the association of DCDC2 and KIAA0319 markers with left temporo-parietal white matter volume (Darki et al. 2012). Left temporo-parietal white matter volumes were then associated with reading skills in the same subjects, suggesting possible mediation between risk genetic markers and behavioral outcome (Darki et al. 2012). In addition to volumetric imaging measures, $D C D C 2$ has also been associated with brain activation patterns during reading-related tasks using functional magnetic resonance imaging (fMRI) (Cope et al. 2012). ACOT13, which may tag variation in KIAA0319, was associated with asymmetry in activation of the superior temporal sulcus during reading tasks (Pinel et al. 2012). The observed lower asymmetry of brain activation patterns was similar to the increased bilateral symmetry in brain activation seen in impaired school-age child readers (Brown et al. 2005). These initial studies have started to provide insight into the neuroimaging implications of behaviorally associated DCDC2 and KIAA0319 markers. However, further study and replication analyses are needed to understand the connection between risk genes, neuroimaging implications, and the ultimate neurobehavioral phenotype.

DYX3 candidate risk genes MRPL19 and GCFC2 are highly co-expressed in brain regions implicated in reading processes, including the inferior frontal and temporal occipital regions as well as the superior temporal, parietal temporal and middle temporal gyri (Anthoni et al. 2007). Furthermore, expression of MRPL19 and GCFC2 are correlated strongly with other RD candidate genes, including $D Y X 1 C 1, D C D C 2$, KIAA0319 and ROBO1 (Anthoni et al. 2007). Associated markers in the MRPL19/GCFC2 locus were related with white matter in the posterior corpus callosum and cingulum, regions that connect large portions of the parietal, occipital and temporal lobes (Scerri et al. 2012). Furthermore, a recent GWAS showed an association of $G C F C 2$ with hippocampal volume (Melville et al. 2012). These initial studies point to MRPL19 and $G C F C 2$ influencing cortical measures and white matter volumes in temporal and hippocampal regions, which then may influence language and neurocognitive traits. However, more work is needed to replicate and substantiate these findings in independent studies.

The overall goal of this study is to examine the neuroimaging implications of DYX2 and DYX3 markers previously associated with reading, language, and/or IQ. To accomplish this, we utilize genetic and neuroimaging data collected in typically developing children in the Pediatric Imaging Neurocognition Genetics (PING) study. First, using the information gained by our recent association scan of the DYX2 locus in an unselected sample, we examine the replicated markers with cortical thickness and volume using structural magnetic resonance imaging (sMRI) and fractional anisotropy (FA) using diffusion tensor imaging (DTI) (Powers et al. 2013; Eicher et al. 2014). Second, we perform association analyses of DYX3 markers previously associated with neuroimaging measures in hippocampal and temporal regions (Scerri et al. 2012; Melville et al. 2012). Here, we aim to confirm the contribution of these markers to neuroimaging phenotypes in these cortical regions.

\section{Methods}

\section{The pediatrics imaging neurocognition genetics (PING) study}

Recruitment and experimental methods for the PING study are described in detail elsewhere, but are summarized briefly below (Akshoomoff et al. 2014; Brown et al. 2012; Fjell et al. 2012; Walhovd et al. 2012; Eicher et al. 2013). The PING study is a cross-sectional cohort of typically developing children between the ages of 3 and 20 years. Subjects were excluded for history of major developmental, psychiatric, and/or neurological disorders, brain injury, or medical conditions that 
affect neurological development. However, subjects were not excluded due to learning or language disabilities such as RD and LI. The human research protections programs and institutional review boards at the 10 institutions (Weil Cornell Medical College, University of California at Davis, University of Hawaii, Kennedy Krieger Institute, Massachusetts General Hospital, University of California at Los Angeles, University of California at San Diego, University of Massachusetts Medical School, University of Southern California, and Yale University) participating in the PING study approved all experimental and consenting procedures. For individuals under 18 years of age, parental informed consent and child assent (for those 7 to 17 years of age) were obtained. All participants age 18 years and older gave their written informed consent.

\section{PING imaging analyses}

PING imaging techniques, data acquisition, and analyses are discussed in depth elsewhere and briefly below (Brown et al. 2012; Fjell et al. 2012; Walhovd et al. 2012). Across the ten sites and 12 scanners, a standardized multiple modality highresolution sMRI protocol was implemented, involving 3D T1and T2-weighted volumes and a set of diffusion-weighted scans. At the University of California at San Diego (UCSD), data were obtained on a GE 3 T SignaHD $\times$ scanner and a $3 \mathrm{~T}$ Discovery $750 \times$ scanner (GE Healthcare) using eight-channel phased array head coils. The protocol included a conventional three-plane localizer, a sagittal 3D inversion recovery spoiled gradient echo T1-weighted volume optimized for maximum gray/white matter contrast (echo time $=3.5 \mathrm{~ms}$, repetition time $=8.1 \mathrm{~ms}$, inversion time $=640 \mathrm{~ms}$, flip angle $=8^{\circ}$, receiver bandwidth $= \pm 31.25 \mathrm{kHz}, \mathrm{FOV}=24 \mathrm{~cm}$, frequency $=256$, phase $=192$, slice thickness $=1.2 \mathrm{~mm}$ ), and two axial 2D DTI pepolar scans (30-directions bvalue $=1000, \mathrm{TE}=83 \mathrm{~ms}, \mathrm{TR}=$ $13,600 \mathrm{~ms}$, frequency $=96$, phase $=96$, slice thickness $=$ $2.5 \mathrm{~mm}$ ). Acquisition protocols with pulse sequence parameters identical or near identical to those protocols used at UCSD were installed on scanners at the other sites. Data were acquired on all scanners to estimate relaxation rates and measure and correct for scanner-specific gradient coil nonlinear warping. Image files in DICOM format were processed with an automated processing stream written in MATLAB (Natick, MA) and C++ by the UCSD Multimodal Imaging Laboratory. T1-weighted structural images were corrected for distortions caused by gradient non-linearities, co-registered, averaged, and rigidly re-sampled into alignment with an atlas brain. Image post-processing and analysis were performed using a fully automated set of tools available in the FreeSurfer software suite (http://surfer.nmr.mgh.harvard.edu/) as well as an atlas-based method for delineating and labeling white matter fiber tracts (Fischl 2012).

\section{DTI methods}

Diffusion-weighted images were corrected for eddy current distortion using a least squares inverse and iterative conjugate gradient descent method to solve for the 12 scaling and translation parameters describing eddy current distortions across the entire diffusion MRI scan, explicitly taking into account the orientations and amplitudes of the diffusion gradient (Zhuang et al. 2006). Head motion was corrected by registering each diffusion-weighted image to a corresponding image synthesized from a tensor fit to the data (Hagler et al. 2009). Diffusion MRI data were corrected for spatial and intensity distortions caused by B0 magnetic field in-homogeneities using the reversing gradient method (Holland et al. 2010). Distortions caused by gradient nonlinearities were corrected by applying a predefined, scanner-specific, nonlinear transformation (Jovicich et al. 2006). Diffusion-weighted images were automatically registered to T1-weighted structural images using mutual information and rigidly re-sampled into a standard orientation relative to the T1-weighted images with isotropic 2-mm voxels (Wells et al. 1996). Cubic interpolation was used for all re-sampling steps. Conventional DTI methods were used to calculate diffusion measures (Basser et al. 1994; Pierpaoli et al. 1996). Scanning duration for the DTI sequence was 4:24 min. White matter fiber tracts were labeled using a probabilistic-atlas based segmentation method (Hagler et al. 2009). Voxels containing primarily gray matter or cerebral spinal fluid, identified using FreeSurfer's automated brain segmentation were excluded from analysis (Fischl et al. 2002). Fiber tract volumes were calculated as the number of voxels with probability greater than 0.08 , the value that provided optimal correspondence in volume between atlasderived regions of interest (ROIs) and manually traced fiber tracts. Next, fractional anisotropy (FA) was calculated within these atlas-derived fiber ROIs for every subject.

\section{Genetics methods in PING}

Subjects were genotyped on the Illumina Human660W-Quad BeadChip (San Diego, CA), with markers passing quality control filters (sample call rate $>98 \%$, SNP call rate $>95 \%$, minor allele frequency $>5 \%$ ). A reference panel for genetic ancestry was constructed as previously described (Brown et al. 2012; Fjell et al. 2012; Walhovd et al. 2012). To assess ancestry and admixture proportions, we used a supervised clustering approach implemented in the ADMIXTURE software and grouped participant data into six clusters corresponding to six major continental populations: African, Central Asian, East Asian, European, Native American, and Oceanic (Alexander et al. 2009; Brown et al. 2012; Fjell et al. 2012; Walhovd et al. 2012). To prevent possible population stratification and as past genetic associations with selected markers were in European populations, only subjects with a 
European genetic ancestry factor (GAF) of 1 were included in analyses.

Fourteen markers previously showed evidence of replicated association with reading-, language-, and/or IQ-related traits within the DYX2 locus (Powers et al. 2013; Eicher et al. 2014); of these, 7 were directly genotyped in the PING study (Table 1). Additionally, rs9461045, a putative functional SNP associated with expression of KIAA0319, was directly genotyped in PING, totaling 8 DYX2 markers for analysis (Dennis et al. 2009). Three DYX3 markers had previously been associated with neuroimaging phenotypes and were directly genotyped in the PING study (Table 1) (Scerri et al. 2012; Melville et al. 2012). Markers were coded as either (1) carriers versus non-carriers of the minor allele (minor allele frequency $<0.25$ ) or $(2)$ homozygous major allele versus heterozygous versus homozygous minor (minor allele frequency $>0.25$ ), termed "Additive" (Table 1).

\section{Imaging-genetics analysis}

Imaging-genetics analyses were performed in individuals of European genetic ancestry $(n=332)$ with imaging measures and DYX2/DYX3 genotypes that passed quality control. Scanner, age, handedness, socioeconomic status, and sex were included as covariates in all analyses (Akshoomoff et al. 2014; Brown et al. 2012; Fjell et al. 2012; Walhovd et al. 2012; Eicher et al. 2013). Different ROIs and imaging modalities were chosen for DYX2 and DYX3 markers (Supplemental Tables 1-2). DYX2 markers were conditioned on FA and cortical thickness in 16 fiber tracts of interest and 15 ROIs, respectively (Supplemental Table 1). Fiber tracts of interest and ROIs were chosen for DYX2 associations based on their previous implications in language and reading. DYX2 markers were examined for fractional anisotropy (FA) in the following fiber tracts: All Fiber Tracts (All), Inferior Longitudinal Fasciculus (ILF), Inferior Fronto-occipital Fasciculus (IFO), Superior Longitudinal Fasciculus (SLF), Temporal Superior Longitudinal Fasciculus (tSLF), Parietal Superior Longitudinal Fasciculus (pSLF), and Striatal Inferior Frontal Cortex (SIFC) in both right and left hemispheres, as well as All Fiber Tracts and Corpus Callosum (CC) bilaterally (Supplemental Table 1). DYX2 cortical ROIs were selected using the genetically relevant cortical parcellations as described in Chen et al. (Chen et al. 2011, 2012). We chose to use these parcellations because they are more likely to show associations with genetic factors based on their previously explored genetic relationships (Chen et al. 2011, 2012). DYX2 markers were examined for cortical thickness in the following regions: Superior Parietal, Orbitofrontal, Superior Temporal, Inferior Parietal, Dorsomedial Frontal, Precuneus, Dorsolateral Prefrontal, Pars Opercularis, and Central in the left hemisphere, as well as Occipital, Anteromedial Temporal, and Posterolateral Temporal in both right and left hemispheres (Supplemental Table 1). Associations with DYX3 markers were conditioned on cortical thickness and volume measures of ROIs derived using the FreeSurfer software suite (http://surfer.nmr.mgh.harvard.edu/) (Supplemental Table 2) (Fischl 2012). DYX3 ROIs were selected to replicate previous associations in hippocampal and temporal cortical regions (Supplemental Table 2).

Associations of genotypes of interest with neuroimaging traits were tested by multiple regression analyses in $\mathrm{R}$ using the PING data portal (https://mmil-dataportal.ucsd.edu). To correct for the multiple ROIs, we set a statistical threshold of 0.05 divided by the number of ROIs tested for each imaging modality. For instance, a threshold of $0.003333(0.05 / 15$ regions of interest) was used for DYX2 associations of cortical thickness. A threshold of $0.003125(0.05 / 16$ regions of
Table 1 DYX3 (Chromosome $2 \mathrm{p} 12, n=3)$ and DYX2 (Chromosome 6p22, $n=8$ ) markers directly genotyped in the PING study that showed replicated association with $\mathrm{RD}$, LI, and/or IQ/

\begin{tabular}{llllllll}
\hline SNP & Ch & BP & $\begin{array}{l}\text { Min } \\
\text { All }\end{array}$ & MAF & Gene & $\begin{array}{l}\text { Associated } \\
\text { traits }\end{array}$ & Coded As: \\
\hline rs917235 & 2 & 75825819 & G & 0.339 & N/A & RD, IQ & Additive \\
rs6732511 & 2 & 75839733 & A & 0.095 & N/A & RD, IQ & Carrier vs. Non-carrier \\
rs2298948 & 2 & 75926565 & G & 0.172 & GCFC2 & Hippocampus & Carrier vs. Non-carrier \\
rs707864 & 6 & 24305848 & G & 0.117 & DCDC2 & RD, LI & Carrier vs. Non-carrier \\
rs9295626 & 6 & 24587339 & A & 0.214 & KIAA0319 & RD, LI, IQ & Carrier vs. Non-carrier \\
rs10456309 & 6 & 24589562 & A & 0.032 & KIAA0319 & RD, LI, IQ & Carrier vs. Non-carrier \\
rs4576240 & 6 & 24596478 & A & 0.117 & KIAA0319 & RD, LI, IQ & Carrier vs. Non-carrier \\
rs9461045 & 6 & 24649061 & A & 0.172 & KIAA0319 & RD, LI, IQ & Carrier vs. Non-carrier \\
rs3777663 & 6 & 24700235 & G & 0.215 & $A C O T 13$ & LI, IQ & Carrier vs. Non-carrier \\
rs3756814 & 6 & 24705835 & C & 0.367 & C6orf62 & LI, IQ & Additive \\
rs9348646 & 6 & 24820219 & G & 0.306 & $F A M 65 B$ & IQ & Additive \\
\hline
\end{tabular}

Ch Chromosome, Min All Minor Allele, MAF Minor Allele Frequency, RD Reading Disability, LI Language impairment

${ }^{a}$ rs917235 and rs6732511 are located in the DYX3 locus upstream of GCFC2 and MRPL19 
interest) was used for associations of DYX2 with FA and DYX3 with cortical thickness and volume. Associations were considered suggestive with $p<0.01$. LD of genetic markers was calculated as D' for all possible pairs of SNPs with Haploview v4.2 (Barrett et al. 2005).

\section{Results}

The results of the genetic associations of DYX2 and DYX3 markers with imaging phenotypes are presented in Table 2 and in Supplemental Tables 3-6. The results from the DYX2 locus are presented first, followed by the DYX3 locus.

\section{The DYX2 locus}

Of the 8 DYX 2 markers analyzed, 3 showed associations with neuroimaging phenotypes: rs9461045 in KIAA0319, rs3777663 in ACOT13, and rs9348646 in FAM65B. In this sample, the LD structure of the DYX2 locus suggests that rs9461045, rs3777663, and rs9348646 may be tagging the same genomic variation (Supplemental Figure 1). The strongest associations were seen with rs9461045 and cortical thickness in the left orbitofrontal region $\left(p=4.89 \times 10^{-4}\right)$ (Table 2). This association between the putative functional marker in KIAA0319, rs9461045, and left orbitofrontal cortical thickness persisted $\left(p=5.00 \times 10^{-3}\right)$ when average overall cortical thickness was included as a covariate in the model, suggesting specific effects of the marker in this region. There was suggestive association of rs 3777663 in ACOT13 with cortical thickness in the left pars opercularis $\left(p=4.64 \times 10^{-3}\right)$ but with no other ROI examined $(p<0.05)$ (Table 2, Supplemental Table 3). This association persisted when overall average cortical thickness was included as a covariate in the model ( $p=$ $4.0 \times 10^{-4}$ ), indicating specific effects of ACOT13 in the left pars opercularis region.

DYX2 associations with FA were suggestive and typically global in nature (Table 2, Supplemental Table 4). The strongest associations were seen with rs9461045 in KIAA0319 and rs9348646 in FAM65B. These included associations of rs9461045 with FA in the corpus callosum (CC, $p=5.89 \times$ $\left.10^{-3}\right)$ as well as rs9348646 with FA in the left superior longitudinal fasciculus (SLF) $\left(p=4.61 \times 10^{-3}\right)$ and right temporal $\operatorname{SLF}(\mathrm{tSLF})\left(p=7.26 \times 10^{-3}\right)$ (Table 2). However, when overall FA was included as a covariate in these models, the associations with FA in these fiber tracts of interest were attenuated ( $p>0.05$ ), indicating that the suggestive effects of KIAA0319 and FAM $65 B$ genotypes reflect global FA effects as opposed to specific regional effects $(\mathrm{p}>0.05)$.

\section{The DYX3 locus}

We examined the association of three DYX3 markers previously associated with various neurocognitive and imaging traits (Table 1). There were suggestive associations with neuroimaging phenotypes in temporal regions with all three
Table 2 Summary of associations $(p<0.01)$ of DYX2 and DYX3 markers with imaging measures

\begin{tabular}{llllll}
\hline ROI & DYX2 markers & Gene/Locus & Measure & Effect & $p$-value \\
Left pars opercularis & rs3777663 & ACOT13 & Thickness & 0.03732 & $4.64 \times 10^{-3}$ \\
Left orbitofrontal & rs9461045 & KIAA0139 & Thickness & -0.0476 & $4.89 \times 10^{-4 b}$ \\
Corpus callosum & rs9461045 & KIAA0139 & FA & -0.00842 & $5.89 \times 10^{-3}$ \\
Right All & rs9348646 & FAM65B & FA & -0.00385 & $9.20 \times 10^{-3}$ \\
Left SLF & rs9348646 & FAM65B & FA & -0.00576 & $4.61 \times 10^{-3}$ \\
Right SLF & rs9348646 & FAM65B & FA & -0.00607 & $7.26 \times 10^{-3}$ \\
Left tSLF & rs9348646 & FAM65B & FA & -0.00651 & $2.10 \times 10^{-3 b}$ \\
Left pSLF & rs9348646 & FAM65B & FA & -0.00527 & $1.00 \times 10^{-2}$ \\
ROI & DYX3 markers & Gene/Locus & Measure & Effect & $p$-value \\
Left middle temporal & rs917235 & DYX3 & Thickness & 0.05897 & $3.96 \times 10^{-3}$ \\
Right Middle Temporal & rs2298948 & GCFC2 & Thickness & 0.03175 & $5.65 \times 10^{-3}$ \\
Right Inferior Temporal & rs2298948 & GCFC2 & Volume & -548.75 & $7.21 \times 10^{-3}$ \\
Right Fusiform & rs6732511 & DYX3 & Volume & 478.22 & $3.15 \times 10^{-3}$ \\
\hline
\end{tabular}

All All Fiber Tracts, SLF Superior Longitudinal Fasiculus, $t S L F$ Temporal Superior Longitudinal Fasiculus, $p S L F$ Parietal Superior Longitudinal Fasiculus

${ }^{\mathrm{a}}$ To correct for multiple testing with DYX2 markers, we used a threshold of 0.00333 for cortical thickness $(0.05$ divided by 15 regions of interest) and 0.003125 for FA ( 0.05 divided by 16 regions of interest). To correct for multiple testing with DYX3 markers, we used a threshold of 0.003125 for cortical thickness $(0.05$ divided by 16 regions of interest) and 0.002778 for cortical volume ( 0.05 divided by 18 regions of interest). Associations deemed suggestive if $p<0.01$

${ }^{\mathrm{b}}$ Survives correction for multiple testing as described above

${ }^{\mathrm{c}}$ rs917235 and rs6732511 are located in the DYX3 locus upstream of GCFC2 and MRPL19 
markers. rs6732511 was in LD with both rs917235 and rs2298948; however, rs917235 and rs2298948 were not in LD with each other (Supplemental Figure 2). rs917235 and rs6732511 showed suggestive association with cortical thickness in the left middle temporal region $\left(p=3.96 \times 10^{-3}\right)$ and cortical volume in the right fusiform region $\left(p=3.15 \times 10^{-3}\right)$, respectively (Table 2, Supplemental Tables 5-6). There were also suggestive associations of rs 2298248 with cortical thickness and volume in various temporal regions, including cortical thickness in the right middle temporal region $(p=5.65 \times$ $10^{-3}$ ) and cortical volume in the right inferior temporal region $\left(p=7.21 \times 10^{-3}\right)$ (Table 2, Supplemental Tables 5-6). Notably, there were no associations of rs2298248 with hippocampal measures, as previously reported in the literature (Melville et al. 2012) (Supplemental Table 6).

\section{Discussion}

The overall goal of this study was to gain biological insight into genetic markers that previously showed replicated associations with reading-, language-, and/or IQ-related traits. To do so, we interrogated markers within the DYX2 and DYX3 loci previously associated with neurobehavioral and neuroimaging traits. Within the DYX2 locus, there were associations of KIAA0319 with cortical thickness in the left orbitofrontal region and $F A M 65 B$ with global FA, with suggestive associations of KIAAO319 with global FA and ACOT13 with cortical thickness in the left pars opercularis region. These results suggest where and how DYX2 risk variants may give rise to their biological effects upon neurocognitive and language development. Additionally, we observed suggestive associations of DYX3 markers with cortical thickness and volume measures in temporal regions, further proposing a possible role of DYX3 risk elements (hypothesized to be MRPL19 and/or $G C F C 2$ ) in temporal regions. Notably, we did not replicate the associations between DYX3 markers and hippocampal volume, failing to confirm the findings of a previous study (Melville et al. 2012).

\section{Associations of DYX2 Genes KIAA0319, ACOT13, and $F A M 65 B$}

Associations of DYX2 markers with cortical thickness and FA were limited to two genomic regions: KIAA0319/ACOT13 and $F A M 65 B$. In our present and previous analyses, markers in the $5^{\prime}$ region of KIAA0319, including rs 9461045 , and rs3777663 in ACOT13 are in high LD with each other and thus, appear to tag the same genomic locus (Eicher et al. 2014). The literature shows the minor allele of rs 9461045 to be the risk allele, while our recent report suggests the minor allele of rs3777663 is protective (Dennis et al. 2009; Eicher et al. 2014). This was possibly mirrored by our imaging- genetic results. KIAA0319 and ACOT13 markers were associated with cortical thickness in the left orbitofrontal and left pars opercularis regions, respectively. The risk minor allele of rs9461045 in KIAA0319 was associated with decreased cortical thickness, and the protective minor allele of rs3777663 in ACOT13 associated with increased cortical thickness. Although the association signals of KIAA0319 and $A C O T 13$ cannot be disentangled in this and other studies, the functional role of KIAA0319 in neuronal migration makes it the likely effector gene (Newbury et al. 2011; Peschansky et al. 2010; Szalkowski et al. 2012, 2013; Centanni et al. 2014). These results, in conjunction with the literature, suggest that risk alleles of KIAA0319 impair neuronal migration, resulting in reduced cortical thickness and then manifesting itself in poorer language and neurocognitive outcomes. Protective alleles, tagged by rs 3777663 , produce the opposite effects with ultimately improved language and neurocognitive skills. Future experimentation is needed to further demonstrate a direct link between genetic variant, neuronal migration, neuroimaging manifestation (in this case, cortical thickness), and the ultimate neurocognitive traits. Additionally, functional molecular work should discern whether ACOT13 plays a role in neural phenotypes, particularly in the left orbitofrontal and pars opercularis regions.

Both KIAA0319 and FAM65B were associated with overall FA, suggesting that risk alleles negatively impact the white matter integrity of fiber tracts. The global effects of these genes on FA suggest that KIAA0319 and FAM65B have substantial impact on how brain circuits integrate various stimuli. In this PING sample, rs9461045 in KIAA0319 and rs9348646 in $F A M 65 B$ were in moderately strong LD with each other $\left(D^{\prime}=0.81\right)$, indicating that these SNPs may be tagging the same genomic variation (Supplemental Figure 1). However, our previous study of a larger sample of European children showed no evidence of LD between KIAA0319 and FAM65B (Eicher et al. 2014). Future studies, particularly of the far less studied $F A M 65 B$, are needed to see how risk variants may influence myelination and/or neurophysiological properties throughout the entire brain.

\section{Suggestive associations of DYX3 markers}

In this study, we show suggestive evidence of association for DYX3 markers with neuroimaging measures in temporal regions. Specifically, there were suggestive relationships between DYX3 markers in the middle and inferior temporal regions, as well as the fusiform gyrus. Previous work showed expression of the two hypothesized DYX3 risk genes, MRPL19 and GCFC2, in these temporal regions (Anthoni et al. 2007). Temporal lobe functions have been well described and include auditory and visual processing, language comprehension, meaning derivation, and formation of new memories. Specifically, functional brain studies on individuals with $\mathrm{RD}$ 
have highlighted decreased activity in the left temporoparietal region during both phonological processing tasks (Shaywitz et al. 1998; Temple et al. 2001) and simple speech tasks, with a notable increased level of activity in corresponding areas in the right brain in children with impaired reading and language skills (Breier et al. 2003; Brown et al. 2014). Additionally, previous work has shown decreased gray matter volume bilaterally in fusiform gyri in adolescents with $\mathrm{RD}$ (Kronbichler et al. 2008). In our present analysis, rs6732511 and rs2298948 were in LD and demonstrated suggestive association with cortical volume in the right fusiform gyrus. The literature shows the minor allele of rs 6732511 to be protective (Anthoni et al. 2007), while the minor allele of rs2298948 has been shown to be the risk allele (Melville et al. 2012). Here, the risk minor allele of rs2298948 was associated with decreased cortical volume possibly yielding adverse language and neurocognitive outcomes, while the protective minor allele of rs6732511 associated with increased cortical volume possibly giving rise to positive language and neurocognitive outcomes. Future studies, using other independent methods, such as animal models and/or longitudinal human neuroimaging strategies, are necessary to confirm a direct relationship between risk marker, neuroimaging observation, and neurocognitive outcome.

Notably, we did not observe associations of rs2298948 with cortical volume measures in the hippocampus, as was observed in a GWAS of hippocampal volume (Melville et al. 2012). The lack of replication of this GWAS signal does not necessarily mean that GCFC2 does not contribute to hippocampal volume, as the present study substantially differs in terms of age (pediatric versus adult populations). Little is known of the possible neural function of the DYX3 candidate genes MRPL19 and GCFC2. In order to further support each of their roles in $\mathrm{RD}, \mathrm{LI}$, and neurocognition, functional and animal-based work should be completed specifically interrogating their cellular and neural contributions.

\section{Use of imaging-genetics in neurocognitive traits}

Currently, we have an incomplete view of the biological etiologies underlying RD, LI, and related neurocognitive traits. Neuropsychological, genetic, molecular, and imaging studies have made much progress into identifying the specific impairments, candidate genes/signatures, and possible pathways that may contribute to the deficits observed in dyslexia and related disorders. However, how these varying levels of phenotype interact and relate to each other to lead to the ultimate neurocognitive phenotype remains elusive. Imaging-genetics studies can suggest a more mechanistic understanding into the pathophysiology of these disorders, and provide an ethical means to gain mechanistic insight into the pathophysiology in human subjects in vivo. Instead of solely relying upon animal models that may approximate the behavioral and biological deficits seen in RD and LI, human imaginggenetics allow for the direct examination of human risk genetic variants with imaging data directly related to reading and language processes. Additionally, neuroimaging traits may represent measureable phenotypes closer to the underlying biology seen in behavior and cognition. Therefore, finding genetic associations with phenotypes closer to the underlying biology, in this case neuroimaging, may be more readily detectable than those conditioned on neurocognitive and language measures (Thompson et al. 2010). The neuroimaging consequences of risk variants can also inform mechanistic studies in regards to where and how neurological dysfunction occurs. However, interactive effects with other genetic and environmental factors must be taken into account to effectively uncover the underlying mechanisms of these traits.

\section{Limitations}

There are limitations in the presented imaging-genetic analyses. First, discerning whether these neuroimaging phenotypes are causal of or resulting from language capabilities is challenging due to the inherent complexity and plasticity of the brain. Further neurophysiological and molecular interrogation using cell-based and organismal models, as well as longitudinal imaging-genetic studies, can help in determining causal and temporal relationships. Second, interpreting what imaging data and their associations with genetic and behavioral factors actually mean in a biological context is challenging. Although there are numerous hypotheses regarding the actual meaning of FA and structural measures through DTI and sMRI, it is still difficult to make definitive conclusions about the biological and behavioral implications of these data. Third, our analyses started with a small subset of genetic variants and brain ROIs. We then examined the implications of these specific genetic markers on brain imaging measures in specific brain regions. Selection bias, along with incomplete coverage of associated genes and the rest of the genome, could lead to misleading and incomplete results and hypotheses. Future studies could use neuroimaging phenotypes as an endophenotype to condition GWAS, sequencing, and voxel-based analyses in order to discover novel genes and neuroimaging traits that contribute to reading, language, and cognition. Additionally, our cross-sectional, unselected sample permitted us only to examine general, quantitative performance as opposed to case-control differences. The lack of case-control analyses may explain the absence of $D C D C 2$ associations, as $D C D C 2$ tends to generally be associated with severe case-control phenotypes (Meng et al. 2005; Powers et al. 2013). Future samples with different recruitment strategies and/or large sample size should examine imaging-genetic associations between these ROIs and markers of interest. Lastly, we limited our sample size to 
those of European ancestry as this study was a direct followup of genetic studies in European samples (Eicher et al. 2014; Scerri et al. 2012). This limited sample size and the allele frequencies of the examined SNPs (Table 1) prevented us for making meaningful inferences on age and gene-by-age effects on brain development as shown in other PING studies (Brown et al. 2012; Douet et al. 2014). Identification of functional SNPs and variants in the DYX2 and DYX3 regions as well as studies of these SNPs in non-European samples will enable us to examine these gene-by-age effects with greater confidence and accuracy.

\section{Conclusion}

In conclusion, this study aimed to interrogate the neuroimaging consequences of genetic markers that had shown replicated associations with reading-, language-, and/or IQ-related traits. In our analyses of the DYX2 locus, we observed associations of KIAA0319, ACOT13, and FAM65B with cortical thickness and/or FA. We also observed suggestive associations of DYX3 markers with cortical thickness and volume measures within temporal regions. These associations offer insight into how these risk genetic markers may give rise to deficits in reading, verbal language, and IQ. Future studies should further interrogate these neurological phenotypes by using cellular, organismal, and molecular models. These studies could further connect risk genetic variants, to cellular phenotypes, to neuroimaging alterations, and to the ultimate deficits in language and communication.

Acknowledgments The authors gratefully thank the children, adolescents, adults, and parents who participated in the PING study. PING data are disseminated by the PING Coordinating Center at the Center for Human Development, University of California at San Diego.

\section{Compliance with Ethical Standards}

Funding Data collection and sharing for this project were funded by the PING Study (National Institutes of Health Grant RC2DA029475). PING is funded by the National Institute on Drug Abuse and the Eunice Kennedy Shriver National Institute of Child Health \& Human Development. This research was specifically funded by the National Institutes of Health (Grant ref: R01 NS043530 and 2P50 HD 02780222 in support of J.R.G., T32 HD07094 awarded to A.M.M., and F31 DC012270 awarded to J.D.E.). In addition, J.D.E. and J.R.G received support from the Manton Foundation.

Conflict of interest John D. Eicher, Angela M. Montgomery, Natacha Akshoomoff, David G. Amaral, Cinnamon S. Bloss, Ondrej Libiger, Nicholas J. Schork, Burcu F. Darst, B. J. Casey, Linda Chang, Thomas Ernst, Jean Frazier, Walter E. Kaufman, Brian Keating, Tal Kenet, David Kennedy, Stewart Mostofsky, Sarah S. Murray, Elizabeth R. Sowell, Hauke Bartsch, Joshua M. Kuperman, Timothy T. Brown, Donald J. Hagler, Jr., Anders M. Dale, Terry L. Jernigan, and Jeffrey R. Gruen declare that they have no conflict of interest.
Ethical approval All procedures performed in studies involving human participants were in accordance with the ethical standards of the institutional and/or national research committee and with the 1964 Helsinki declaration and its later amendments or comparable ethical standards. This article does not contain any studies with animals performed by any of the authors.

Informed consent Informed consent was obtained from all individual participants included in the study.

Open Access This article is distributed under the terms of the Creative Commons Attribution 4.0 International License (http:// creativecommons.org/licenses/by/4.0/), which permits unrestricted use, distribution, and reproduction in any medium, provided you give appropriate credit to the original author(s) and the source, provide a link to the Creative Commons license, and indicate if changes were made.

\section{References}

Akshoomoff, N., Newman, E., Thompson, W. K., McCabe, C., Bloss, C. S., Chang, L., Amaral, D. G., Casey, B. J., Ernst, T. M., Frazier, J. A., Gruen, J. R., Kaufmann, W. E., Kenet, T., Kennedy, D. N., Libiger, O., Mostofsky, S., Murray, S. S., Sowell, E. R., Schork, N., Dale, A. M., \& Jernigan, T. L. (2014). The NIH toolbox cognition battery: results from a large normative developmental sample (PING). Neuropsychology, 28(1), 1-10.

Alexander, D. H., Novembre, J., \& Lange, K. (2009). Fast model-based estimation of ancestry in unrelated individuals. Genome Research, 19(9), 1655-1664.

Anthoni, H., Zucchelli, M., Matsson, H., Müller-Myhsok, B., Fransson, I., Schumacher, J., Massinen, S., Onkamo, P., Warnke, A., Griesemann, H., Hoffmann, P., Nopola-Hemmi, J., Lyytinen, H., Schulte-Körne, G., Kere, J., Nöthen, M. M., \& Peyrard-Janvid, M. (2007). A locus on 2p12 containing the co-regulated MRPL19 and C2orf3 genes is associated to dyslexia. Human Molecular Genetics, 16(6), 667-677.

Barrett, J. C., Fry, B., Maller, J., \& Daly, M. J. (2005). Haploview: analysis and visualization of LD and haplotype maps. Bioinformatics, 21, 263-265.

Basser, P. J., Mattiello, J., \& LeBihan, D. (1994). MR diffusion tensor spectroscopy and imaging. Biophysical Journal, 66(1), 259-267.

Bishop, D. V., \& Hayiou-Thomas, M. E. (2008). Heritability of specific language impairment depends on diagnostic criteria. Genes, Brain, and Behavior, 7(3), 365-372.

Breier, J. I., Simos, P. G., Fletcher, J. M., Castillo, E. M., Zhang, W., \& Papanicolaou, A. C. (2003). Abnormal activation of temporoparietal language areas during phonetic analysis in children with dyslexia. Neuropsychology, 17(4), 610-621.

Brown, T. T., Lugar, H. M., Coalson, R. S., Miezin, F. M., Petersen, S. E., $\&$ Schlagger, B. L. (2005). Developmental changes in human cerebral functional organization for word generation. Cerebral Cortex, 15(3), 275-290.

Brown, T. T., Kuperman, J. M., Chung, Y., Erhart, M., McCabe, C., Hagler, D. J., Jr., Venkatraman, V. K., Akshoomoff, N., Amaral, D. G., Bloss, C. S., Casey, B. J., Chang, L., Ernst, T. M., Frazier, J. A., Gruen, J. R., Kaufmann, W. E., Kenet, T., Kennedy, D. N., Murray, S. S., Sowell, E. R., Jernigan, T. L., \& Dale, A. M. (2012). Neuroanatomical assessment of biological maturity. Current Biology, 22(18), 1693-1698.

Brown, T. T., Erhart, M., Avesar, D., Dale, A. M., Halgren, E., \& Evans, J. L. (2014). Atypical right hemisphere specialization for object 
representations in an adolescent with specific language impairment. Frontiers in Human Neuroscience, 8, 82.

Cardon, L. R., Smith, S. D., Fulker, D. W., Kimberling, W. J., Pennington, B. F., \& DeFries, J. C. (1994). Quantitative trait locus for reading disability on chromosome 6. Science, 266(5183), 276-279.

Centanni, T. M., Booker, A. B., Sloan, A. M., Chen, F., Maher, B. J., Carraway, R. S., Khodaparast, N., Rennaker, R., LoTurco, J. J., \& Kilgard, M. P. (2014). Knockdown of the dyslexia-associated gene Kiaa0319 impairs temporal responses to speech stimuli in rat primary auditory cortex. Cerebral Cortex, 24(7), 1753-1766.

Chen, C. H., Panizzon, M. S., Eyler, L. T., Jernigan, T. L., Thompson, W., Fennema-Notestine, C., Jak, A. J., Neale, M. C., Hamza, S., Lyons, M. J., Grant, M. D., Fischl, B., Seidman, L. J., Tsuang, M. T., Kremen, W. S., \& Dale, A. M. (2011). Genetic influences on cortical regionalization in the human brain. Neuron, 72(4), 537-544.

Chen, C. H., Guiterrez, E. D., Thompson, W., Panizzon, M. S., Jernigan, T. L., Eyler, L. T., Fennema-Notestine, C., Jak, A. J., Neale, M. C., Franz, C. E., Lyons, M. J., Grant, M. D., Fischl, B., Seidman, L. J., Tsuang, M. T., Kremen, W. S., \& Dale, A. M. (2012). Hierarchical organization of human cortical surface area. Science, 335(6076), $1634-1636$

Cope, N., Eicher, J. D., Meng, H., Gibson, C. J., Hager, K., Lacadie, C., Fulbright, R. K., Constable, R. T., Page, G. P., \& Gruen, J. R. (2012). Variants in the DYX2 locus are associated with altered brain activation on reading-related brain regions in subjects with reading disability. NeuroImage, 63(1), 148-156.

Darki, F., Peyrard-Janvid, M., Matsson, H., Kere, J., \& Klingberg, T. (2012). Three dyslexia susceptibility genes, DYX1C1, DCDC2, and KIAA0319, affect temporo-parietal white matter structure. Biological Psychiatry, 72(8), 671-676.

Deffenbacher, K. E., Jenyon, J. B., Hoover, D. M., Olson, R. K., Pennington, B. F., DeFries, J. C., \& Smith, S. D. (2004). Refinement of the 6p21.3 quantitative trait locus influencing dyslexia: linkage and association analysis. Human Genetics, 115(2), 128138.

DeFries, J. C., Fulker, D. W., \& LaBuda, M. C. (1987). Evidence for a genetic aetiology in reading disability of twins. Behavior Genetics, 17(3), 263-277.

Dennis, M. Y., Paracchini, S., Scerri, T. S., Prokunina-Olsson, L., Knight, J. C., Wade-Martins, R., Coggill, P., Beck, S., Green, E. D., \& Monaco, A. P. (2009). A common variant associated with dyslexia reduces expression of the KIAA0319 gene. PLoS Genetics, 5, e1000436.

Douet, V., Chang, L., Pritchett, A., Lee, K., Keating, B., Bartsch, H., Jernigan, T. L., Dale, A., Akshoomoff, N., Murray, S., Bloss, C., Kennedy, D. N., Amaral, D., Gruen, J., Kaufmann, W. E., Casey, B. J., Sowell, E., \& Ernst, T. (2014). Schizophrenia-risk variant rs6994992 in the neuregulin-1 gene on brain development trajectories in typically developing children. Translational Psychiatry, 4, e392.

Eicher, J. D., \& Gruen, J. R. (2013). Imaging-genetics in dyslexia: connecting risk genetic variants to brain neuroimaging and ultimately to reading impairments. Molecular Genetics and Metabolism, $110(3), 201-212$

Eicher, J. D., Powers, N. R., Miller, L. L., Akshoomoff, N., Amaral, D. G., Bloss, C. S., Libiger, O., Schork, N. J., Darst, B. F., Casey, B. J., Chang, L., Ernst, T., Frazier, J., Kaufmann, W. E., Keating, B., Kenet, T., Kennedy, D., Mostofsky, S., Murray, S. S., Sowell, E. R., Bartsch, H., Kuperman, J. M., Brown, T. T., Hagler, D. J., Jr., Dale, A. M., Jernigan, T. L., St Pourcain, B., Davey Smith, G., Ring, S. M., Gruen, J. R., \& Pediatric Imaging, Neurocognition, and Genetics Study. (2013). Genome-wide association study of shared components of reading disability and language impairment. Genes, Brain, and Behavior, 12(8), 792-801.

Eicher, J. D., Powers, N. R., Miller, L. L., Mueller, K. L., Mascheretti, S., Marino, C., Willcutt, E. G., DeFries, J. C., Olson, R. K., Smith, S.
D., Pennington, B. F., Tomblin, J. B., Ring, S. M., \& Gruen, J. R. (2014). Characterization of the DYX2 locus on chromosome 6p22 with reading disability, language impairment, and IQ. Human Genetics, 133(7), 869-881.

Fagerheim, T., Raeymaekers, P., Tønnessen, F. E., Pedersen, M., Tranebjaerg, L., \& Lubs, H. A. (1999). A new gene (DYX3) for dyslexia is located on chromosome 2. Journal of Medical Genetics, 36(9), 664-669.

Fischl, B. (2012). FreeSurfer. NeuroImage, 62(2), 774-781.

Fischl, B., Salat, D. H., Busa, E., Albert, M., Dieterich, M., Haselgrove, C., van der Kouwe, A., Killiany, R., Klaveness, S., Montillo, A., Makris, N., Rosen, B., \& Dale, A. M. (2002). Whole brain segmentation: automated labeling of neuroanatomical structures in the human brain. Neuron, 33(3), 41-55.

Fjell, A. M., Walhovd, K. B., Brown, T. T., Kuperman, J. M., Chung, Y., Hagler, D. J., Jr., Venkatraman, V., Roddey, J. C., Erhart, M., McCabe, C., Akshoomoff, N., Amaral, D. G., Bloss, C. S., Libiger, O., Darst, B. F., Schork, N. J., Casey, B. J., Chang, L., Ernst, T. M., Gruen, J. R., Kaufmann, W. E., Kenet, T., Frazier, J., Murray, S. S., Sowell, E. R., van Zijl, P., Mostofsky, S., Jernigan, T. L., Dale, A. M., \& Pediatric Imaging, Neurocognition, and Genetics Study. (2012). Multimodal imaging of the self-regulating developing brain. Proceedings of the National Academy of Sciences of the United States of America, 109(48), 19620-19625.

Francks, C., Paracchini, S., Smith, S. D., Richardson, A. J., Scerri, T. S., Cardon, L. R., Marlow, A. J., MacPhie, I. L., Walter, J., Pennington, B. F., Fisher, S. E., Olson, R. K., DeFries, J. C., Stein, J. F., \& Monaco, A. P. (2004). A 77-kilobase region of chromosome $6 \mathrm{p} 22.2$ is associated with dyslexia in families from the United Kingdom and from the United States. American Journal of Human Genetics, 75, 1046-1058.

Gayán, J., Smith, S. D., Cherny, S. S., Cardon, L. R., Fulker, D. W., Brower, A. M., Olson, R. K., Pennington, B. F., \& DeFries, J. C. (1999). Quantitative-trait locus for specific language and reading deficits on chromosome 6p. American Journal of Human Genetics, 64(1), 157-164.

Graham, S. A., \& Fisher, S. E. (2013). Decoding the genetics of speech and language. Current Opinion in Neurobiology, 23(1), 43-51.

Hagler, D. J., Jr., Ahmadi, M. E., Kuperman, J., Holland, D., McDonald, C. R., Halgren, E., \& Dale, A. M. (2009). Automated white-matter tractography using a probabilistic diffusion tensor atlas: application to temporal lobe epilepsy. Human Brain Mapping, 30(5), 1535-1547.

Harold, D., Paracchini, S., Scerri, T., Dennis, M., Cope, N., Hill, G., Moskvina, V., Walter, J., Richardson, A. J., Owen, M. J., Stein, J. F., Green, E. D., O’Donovan, M. C., Williams, J., \& Monaco, A. P. (2006). Further evidence that the KIAA0319 gene confers susceptibility to developmental dyslexia. Molecular Psychiatry, 11(12), 1085-1091.

Holland, D., Kuperman, J. M., \& Dale, A. M. (2010). Efficient correction of inhomogeneous static magnetic field-induced distortion in echo planar imaging. NeuroImage, 50(1), 175-183.

Jamadar, S., Powers, N. R., Meda, S. A., Gelernter, J., Gruen, J. R., \& Pearlson, G. D. (2011). Genetic influences of cortical gray matter in language-related regions in healthy controls and schizophrenia. Schizophrenia Research, 129(2-3), 141-148.

Jovicich, J., Czanner, S., Greve, D., Haley, E., van der Kouew, A., Gollub, R., Kennedy, D., Schimitt, F., Brown, G., Macfall, J., Fischl, B., \& Dale, A. (2006). Reliability in multi-site structural MRI studies: effects of gradient non-linearity correction on phantom and human data. NeuroImage, 30(2), 436-443.

Kaminen, N., Hannula-Jouppi, K., Kestilä, M., Lahermo, P., Muller, K., Kaaranen, M., Myllyluoma, B., Voutilainen, A., Lyytinen, H., Nopola-Hemmi, J., \& Kere, J. (2003). A genome scan for developmental dyslexia confirms linkage to chromosome $2 \mathrm{p} 11$ and suggests a new locus on 7q32. Journal of Medical Genetics, 40(5), 340-345.

Kaplan, D. E., Gayán, J., Ahn, J., Won, T. W., Pauls, D., Olson, R. K., DeFries, J. C., Wood, F., Pennington, B. F., Page, G. P., Smith, S. D., 
\& Gruen, J. R. (2002). Evidence for linkage and association with reading disability on 6p21.3-22. American Journal of Human Genetics, 70(5), 1287-1298.

Kronbichler, M., Wimmer, H., Staffen, W., Hutzler, F., Mair, A., \& Ladurner, G. (2008). Developmental dyslexia: gray matter abnormalities in the occipitotemporal cortex. Human Brain Mapping, 29(5), 613-625.

Meda, S. A., Gelernter, J., Gruen, J. R., Calhoun, V. D., Meng, H., Cope, N. A., \& Pearlson, G. D. (2008). Polymorphism of DCDC2 reveals differences in cortical morphology of healthy individuals-a preliminary voxel based morphometry study. Brain Imaging and Behavior, 2(1), 21-26.

Melville, S. A., Buros, J., Parrado, A. R., Vardarajan, B., Logue, M. W., Shen, L., Risacher, S. L., Kim, S., Jun, G., DeCarli, C., Lunetta, K. L., Baldwin, C. T., Saykin, A. J., Farrer, L. A., \& Alzheimer's Disease Neuroimaging Initiative. (2012). Multiple loci influencing hippocampal degeneration identified by genome scan. Annals of Neurology, 72(1), 65-75.

Meng, H., Smith, S. D., Hager, K., Held, M., Liu, J., Olson, R. K., Pennington, B. F., DeFries, J. C., Gelernter, J., O'Reilly-Pol, T., Somlo, S., Skudlarski, P., Shaywitz, S. E., Shaywitz, B. A., Marchione, K., Wang, Y., Paramasivam, M., LoTurco, J. J., Page, G. P., \& Gruen, J. R. (2005). DCDC2 is associated with reading disability and modulates neuronal development in the brain. Proceedings of the National Academy of Sciences of the United States of America, 102, 17053-17058.

Newbury, D. F., Fisher, S. E., \& Monaco, A. P. (2010). Recent advances in the genetics of language impairment. Genome Medicine, 2(1), 6.

Newbury, D. F., Paracchini, S., Scerri, T. S., Winchester, L., Addis, L., Richarson, A. J., Walter, J., Stein, J. F., Talcott, J. B., \& Monaco, A. P. (2011). Investigation of dyslexia and SLI risk variants in readingand language-impaired subjects. Behavior Genetics, 41(1), 90-104.

Paracchini, S., Thomas, A., Castro, S., Lai, C., Paramasivam, M., Wang, Y., Keating, B. J., Taylor, J. M., Hacking, D. F., Scerri, T., Francks, C., Richardson, A. J., Wade-Martins, R., Stein, J. F., Knight, J. C., Copp, A. J., LoTurco, J., \& Monaco, A. P. (2006). The chromosome 6 p22 haplotype associated with dyslexia reduces the expression of KIAA0319, a novel gene involved in neuronal migration. Human Molecular Genetics, 15, 1659-1666.

Paracchini, S., Ang, Q. W., Stanley, F. J., Monaco, A. P., Pennell, C. E., \& Whitehouse, A. J. (2011). Analysis of dyslexia candidate genes in the Raine cohort representing the general Australian population. Genes, Brain, and Behavior, 10(2), 158-165.

Pennington, B. F., \& Bishop, D. V. M. (2009). Relations among speech, language, and reading disorders. Annual Review of Psychology, 60, 283-306.

Peschansky, V. J., Burbridge, T. J., Volz, A. J., Fiondella, C., WissnerGross, Z., Galaburda, A. M., LoTurco, J. J., \& Rosen, G. D. (2010). The effect of variation in expression of the candidate dyslexia susceptibility gene homolog Kiaa0319 on neuronal migration and dendritic morphology in the rat. Cerebral Cortex, 20(4), 884-897.

Peyrard-Janvid, M., Anthoni, H., Onkamo, P., Lahermo, P., Zucchelli, M., Kaminen, N., Hannula-Jouppi, K., Nopola-Hemmi, J., Voutilainen, A., Lyytinen, H., \& Kere, J. (2004). Fine mapping of the 2p11 dyslexia locus and exclusion of TACr1 as a candidate gene. Human Genetics, 114(5), 510-516.

Pierpaoli, C., Jezzard, P., Basser, P. J., Barnett, A., \& Di Chiro, G. (1996). Diffusion tensor MR imaging of the human brain. Radiology, 201(3), 637-648.

Pinel, P., Fauchereau, F., Moreno, A., Barbot, A., Lathrop, M., Zelenika, D., Le Bihan, D., Poline, J. B., Bourgeron, T., \& Dehaene, S. (2012). Genetics variants of FOXP2 and KIAA0319/TTRAP/THEM2 locus are associated with altered brain activation in distinct languagerelated regions. Journal of Neuroscience, 32(3), 817-825.

Powers, N. R., Eicher, J. D., Butter, F., Kong, Y., Miller, L. L., Ring, S. M., Mann, M., \& Gruen, J. R. (2013). Alleles of a polymorphic
ETV6 binding site in DCDC2 confer risk of reading and language impairment. American Journal of Human Genetics, 93(1), 19-28.

Scerri, T. S., Morris, A. P., Buckingham, L. L., Newbury, D. F., Miller, L. L., Monaco, A. P., Bishop, D. V., \& Paracchini, S. (2011). DCDC2, KIAA0319, and CMIP are associated with reading-related traits. Biological Psychiatry, 70(3), 237-245.

Scerri, T. S., Darki, F., Newbury, D. F., Whitehouse, A. J., PeyrardJanvid, M., Matsson, H., Ang, Q. W., Pennell, C. E., Ring, S., Stein, J., Morris, A. P., Monaco, A. P., Kere, J., Talcott, J. B., Kingberg, T., \& Paracchini, S. (2012). The dyslexia candidate locus on $2 \mathrm{p} 12$ is associated with general cognitive ability and white matter structure. PLoS One, 7(11), e50312.

Schumacher, J., Anthoni, H., Dahdouh, F., König, I. R., Hillmer, A. M., Kluck, N., Manthey, M., Plume, E., Warnke, A., Remschmidt, H., Hülsmann, J., Cichon, S., Lindgren, C. M., Propping, P., Zucchelli, M., Ziegler, A., Peyrard-Janvid, M., Schulte-Körne, G., Nöthen, M. M., \& Kere, J. (2006). Strong genetic evidence of DCDC2 as a susceptibility gene for dyslexia. American Journal of Human Genetics, 78(1), 52-62.

Shaywitz, S. E., Shaywitz, B. A., Pugh, K. R., Fulbright, R. K., Constable, R. T., Mencl, W. E., Shankweiler, D. P., Liberman, A. M., Skudlarski, P., Fletcher, J. M., Katz, L., Marchione, K. E., Lacadie, C., Gatenby, C., \& Gore, J. C. (1998). Functional disruption in the organization of the brain for reading in dyslexia. Proceedings of the National Academy of Sciences of the United States of America, 95(5), 2636-2641.

Smith, S. D., Pennington, B. F., Boada, R., \& Shriberg, L. D. (2005). Linkage of speech sound disorder to reading disability loci. Journal of Child Psychology and Psychiatry, 46(1), 1057-1066.

Szalkowski, C. E., Fiondella, C. G., Galaburda, A. M., Rosen, G. D., Loturco, J. J., \& Fitch, R. H. (2012). Neocortical disruption and behavioral impairments in rats following in utero RNAi of candidate dyslexia risk gene Kiaa0319. International Journal of Developmental Neuroscience, 30(4), 293-302.

Szalkowski, C. E., Fiondella, C. F., Truong, D. T., Rosen, G. D., LoTurco, J. J., \& Fitch, R. H. (2013). The effects of Kiaa0319 knockdown on cortical and subcortical anatomy in male rats. International Journal of Developmental Neuroscience, 31(2), 116-122.

Temple, E., Poldrack, R. A., Salidis, J., Deutsch, G. K., Tallal, P., Merzenich, M. M., \& Gabrieli, J. D. (2001). Disrupted neural responses to phonological and orthographic processing in dyslexic children: an fMRI study. Neuroreport, 12(2), 299-307.

Thompson, P. M., Martin, N. G., \& Wright, M. J. (2010). Imaging genomics. Current Opinion in Neurology, 23(4), 368-373.

van Soelen, I. L., Brouwer, R. M., van Leeuwen, M., Kahn, R. S., Hulshoff Pol, H. E., \& Boomsma, D. I. (2011). Heritability of verbal and performance intelligence in a pediatric longitudinal sample. Twin Research and Human Genetics, 14(2), 119-128.

Walhovd, K. B., Fjell, A. M., Brown, T. T., Kuperman, J. M., Chung, Y., Hagler, D. J., Jr., Roddey, J. C., Erhart, M., McCabe, C., Akshoomoff, N., Amaral, D. G., Bloss, C. S., Libiger, O., Schork, N. J., Darst, B. F., Casey, B. J., Chang, L., Ernst, T. M., Frazier, J., Gruen, J. R., Kaufmann, W. E., Murray, S. S., van Zijl, P., Mostofsky, S., Dale, A. M., \& Pediatric Imaging Neurocogntion and Genetics Study. (2012). Long-term influence of normal variation in neonatal characteristics on human brain development. Proceedings of the National Academy of Sciences of the United States of America, 109(49), 20089-20094.

Wells, W. M., 3rd, Viola, P., Atsumi, H., Nakajima, S., \& Kikinis, R. (1996). Multi-modal volume registration by maximization of mutual information. Medical Image Analysis, 1(1), 35-51.

Zhuang, J., Hrabe, J., Kangarlu, A., Xu, D., Bansal, R., Branch, C. A., \& Peterson, B. S. (2006). Correction of eddy-current distortions in diffusion tensor images using the known directions and strengths of diffusion gradients. Journal of Magnetic Resonance Imaging, 24(5), 1188-1193. 